Textures and Microstructures, 1988, Vols. 8 \& 9, pp. 401-412

Reprints available directly from the publisher

Photocopying permitted by license only

(C) 1988 Gordon and Breach Science Publishers Inc.

Printed in the United Kingdom

\title{
On the Mechanism of Secondary Recrystallization in Grain Oriented Silicon Iron
}

\author{
G. ABBRUZZESE, I. CIANCAGLIONI and A. CAMPOPIANO \\ Centro Sviluppo Materiali, Roma, Italy
}

(Received August 7, 1987)

\section{Dedicated to the memory of Professor Günter Wassermann}

The final Goss texture in grain oriented silicon iron develops by a secondary recrystallization process in presence of second phase particles.

A sheet of $\mathrm{Fe}-3 \% \mathrm{Si}$ was annealed in a gradient furnace in order to establish the exact temperature of the beginning of abnormal growth.

Measurements of grain size distributions of the different orientations and in particular of the $\{110\}\langle 001\rangle$ grains (within a cone of $\pm 10^{\circ}$ width around the rolling direction) were performed at different stages of grain growth. The so identified Goss grains show a different growth kinetic in comparison with the matrix. The results are analysed and discussed in the framework of the statistical model of grain growth assuming a texture selection process operating.

The agreement between experimental and theoretical results is quite good and allows to state that the main mechanism for the secondary recrystallization in silicon iron is a selective process of grain growth which is controlled by the presence of an inhibitor (dispersion of second phase particles). As a consequence an initial larger grain size for $\{110\}\langle 001\rangle$ oriented grains is not a necessary condition to get the final Goss texture.

KEY WORDS: Secondary recrystallization, continuous grain growth, computer simulation, grain size measurements, orientation determination, etch pits, silicon iron 


\section{INTRODUCTION}

It is well known that the metallurgical mechanism by which Goss texture $(110)\langle 001\rangle$ develops in industrial $\mathrm{Fe}-3 \% \mathrm{Si}$ is a secondary recrystallization process, that occurs in presence of an inhibitor of grain growth (second phase particles or foreign atoms in solid solution) by a sudden size increase of very few grains which consume the whole polycrystalline matrix.

This physical phenomenon has been interpreted for a long time by different models mainly as regards Goss grains nucleation preceding the full recrystallization.

The discussion was principally concentrated on the mechanism by which Goss grains achieve size advantage in the system, because there is a general agreement on the largest driving force for grain growth belonging to the biggest grains in the matrix.

A first explanation is based on the general idea of a faster growth of Goss oriented nuclei during primary recrystallization, leading a certain number of these grains to have larger size in the matrix (May, 1958). A second point of view assumes a preferential coalescence mechanism in clusters of Goss oriented grains because of their very low relative misorientation (Matsuo et al., 1981, Inokuti et al., 1981).

At last a selective process due to a special orientation relationship between Goss and matrix grains has been claimed to act as the main cause of the secondary recrystallization (Koh and Dunn, 1960, Dunn and Mac-Hargue, 1960, Harase et al., 1986, Abbruzzese and Barisoni, 1986). Here, to study the details of grain growth in $\mathrm{Fe}-3 \% \mathrm{Si}$, a typical material with particles of $\left(\mathrm{Mn}, \mathrm{Cu}_{2}\right) \mathrm{S}$ as grain growth inhibitor was heat-treated, simulating the usual industrial annealing process.

The incubation of secondary recrystallization has been observed at different stages of development by optical metallography which gives information on single grain orientation, as X-ray do on the relative intensity of different texture components.

Moreover, based on the statistical model of grain growth (Abbruzzese 1985, Abbruzzese and Lücke 1986a, b, Abbruzzese 1985, Abbruzzese and Lücke 1987) a series of computer simulations were performed to interpret the experimental data in terms of selective grain growth in presence of inhibition from second phase particles. 


\section{EXPERIMENTAL RESULTS}

After primary recrystallization a sheet $(300 \mathrm{~mm}$ length, $30 \mathrm{~mm}$ width, $0.23 \mathrm{~mm}$ thickness) of $\mathrm{Fe}-3 \% \mathrm{Si}$ (see Table 1) was annealed in a temperature gradient furnace simulating the same industrial heating rate $\left(30^{\circ} \mathrm{C} / \mathrm{h}\right.$ up to $930^{\circ} \mathrm{C}$ and 1 hour of soaking time follows) to ascertain the exact temperature (by the position on the sheet), where large grains start to appear indicating the presence of abnormal growth.

Just below that position, measurement of grain size distributions of different orientations (by etch pitting method (Beguinot and Lesbats 1977)) were carried out.

Moreover two other series of measurements were performed at lower temperatures, namely after primary recrystallization $\left(830^{\circ} 3^{\prime}\right.$ in a wet atmosphere for the decarburization of the steel) and at intermediate temperature $\left(855^{\circ} \mathrm{C}\right)$.

By "etch pitting" technique and QTM analysis grain size distributions for the $\{111\}\langle 112\rangle,\{111\}\langle 110\rangle,\{110\}\langle 001\rangle,\{100\}\langle 110\rangle$ and $\{100\}\langle 001\rangle$ orientations and $\{110\}$ planes, without Goss orientation, were evaluated.

The $\{110\}$ grains with $\langle 001\rangle$ direction disoriented 10 degrees as a maximum angle all around the rolling direction were considered Goss grains. The same integration around the exact crystallographic position was performed for the other measured orientations.

About 2000 grains for each annealing temperature were measured and the Goss grains were found to be nearly $15 \%$ of the total number after primary recrystallization and decarburation process.

All the grain size distributions of the different orientation measured on the plane were transformed into volume distributions (Underwood, 1968) assuming for each measured grain an equivalent circle and using the equivalent radius to get the volume transformed distribution.

In Figure 1 the mean radii for all measured texture components

Table 1 Chemical composition (wt $\%$ )

\begin{tabular}{llll}
\hline $\mathrm{C}$ & $\mathrm{Mn}$ & $\mathrm{S}$ & $\mathrm{Cu}$ \\
\hline 0.035 & 0.07 & 0.025 & 0.15 \\
\hline
\end{tabular}




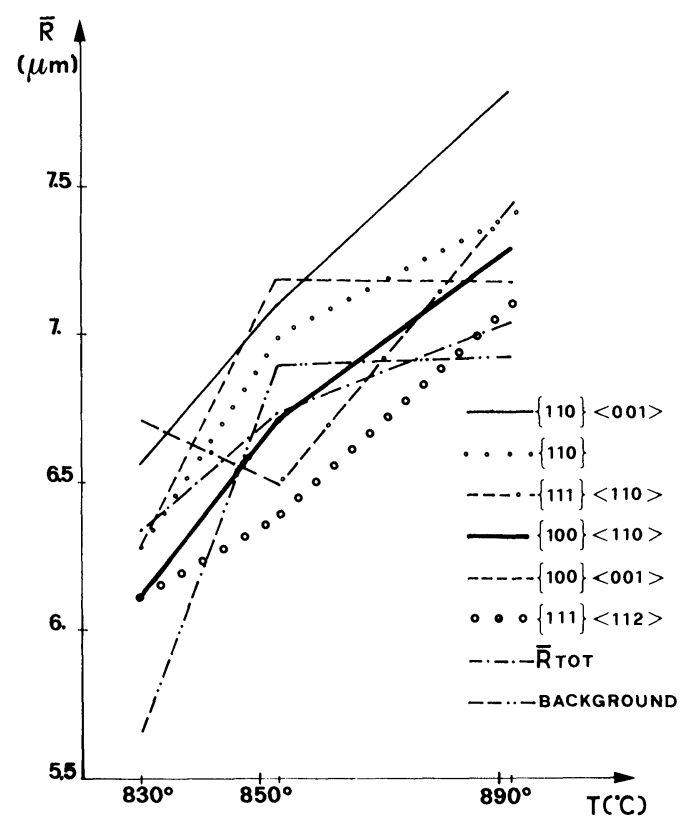

Figure 1 Mean radii of the texture components vs. annealing temperature.

as a function of soaking temperature $\left(830^{\circ}, 853^{\circ}\right.$ and $\left.892^{\circ}\right)$ are shown.

The total mean radius of the system shows a relatively low change during the incubation time before the sudden secondary recrystallization starts $\left(R=6.3 \mu \mathrm{m}\right.$ at $830^{\circ} ; R=7.4 \mu \mathrm{m}$ at $\left.892^{\circ}\right)$, suggesting that the process involves some particular features, which global parameters, as the mean radii, do not point out.

In fact in the secondary recrystallization process of silicon-iron about one among $10^{7}-10^{8}$ grains achieves the final large size $(1-20 \mathrm{~mm})$.

However in Figure 1 the Goss grains, which initially do not have the largest mean radii in the system, appear to grow a little faster than the other orientations that behave more closely to the total mean radius.

The intermediate fall in the plot of $\{111\}\langle 110\rangle$ mean radius is probably due to the difficulties found in the grain classification for the different conditions of image quality obtained in etch pitting 
analysis. In this respect also the behaviour of the "background" component has to be considered partially anomalous. In this component all the grains that could not be classified because their etch pits were corresponding to an intermediate orientation with respect to those chosen, were inserted.

Moreover, the size distributions belonging to the single orientation shown in Figure 1, do not behave much differently from the overall size distribution and again only the Goss orientation shows a significant anomalous behaviour. However the details on the evolution of each size distribution will be discussed elsewhere.

In conclusion it is reasonable to analyze the grain growth process characterizing the overall grain size distribution by only two size distributions belonging to different texture components: one is the Goss orientation, measured with the above-discussed approximation, whereas the other one that we call "matrix", contains all the other grains irrespectively of their orientation.

In Figure 2a the size distribution of the matrix and that of the Goss orientation are compared at the temperature of $830^{\circ} \mathrm{C}$ which represents the condition both after primary recrystallization and a certain grain growth during the decarburization process. Both distributions are here normalized to have 100 as frequency sum.

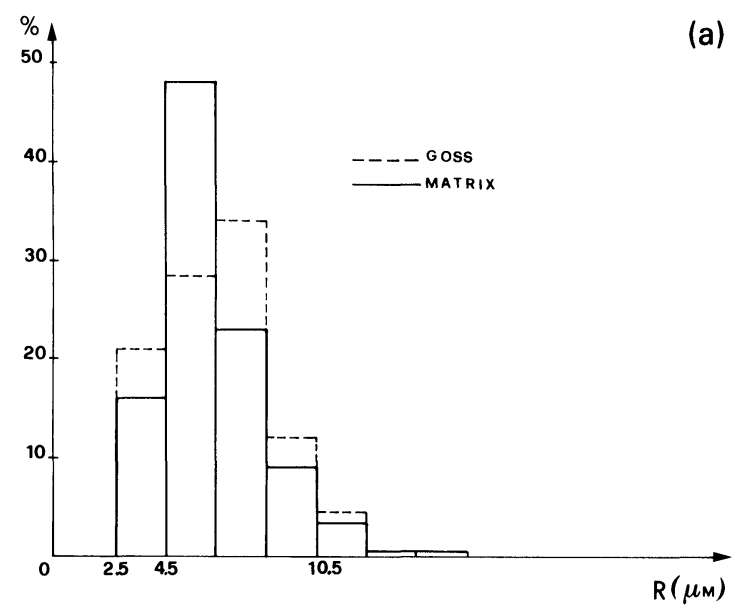

Figure 2 (a) Experimental data of Goss and matrix grain size distributions after primary recrystallization $\left(830^{\circ} 3^{\prime}\right)$. 

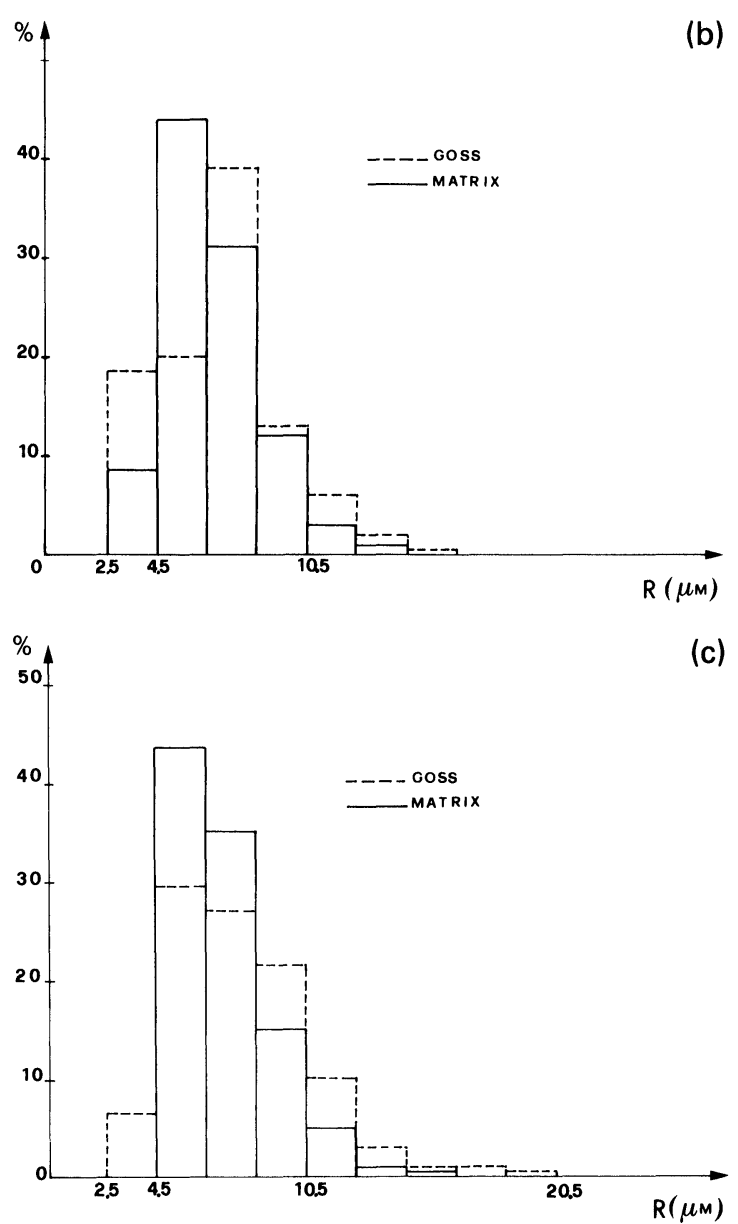

Figure 2 (b), (c) The same kind of experimental size distributions after annealing at $855^{\circ}$ and $892^{\circ}$ respectively.

The average size of Goss grains is a little larger than that of the matrix but the tail of large grains for Goss distribution is shorter.

Obviously comparing the details of the size distribution the relative difference in the number of the measured grains should be taken into account ( $\sim 300$ of Goss oriented grains against $\sim 1700$ as matrix grains).

However these and other measurements already carried out 

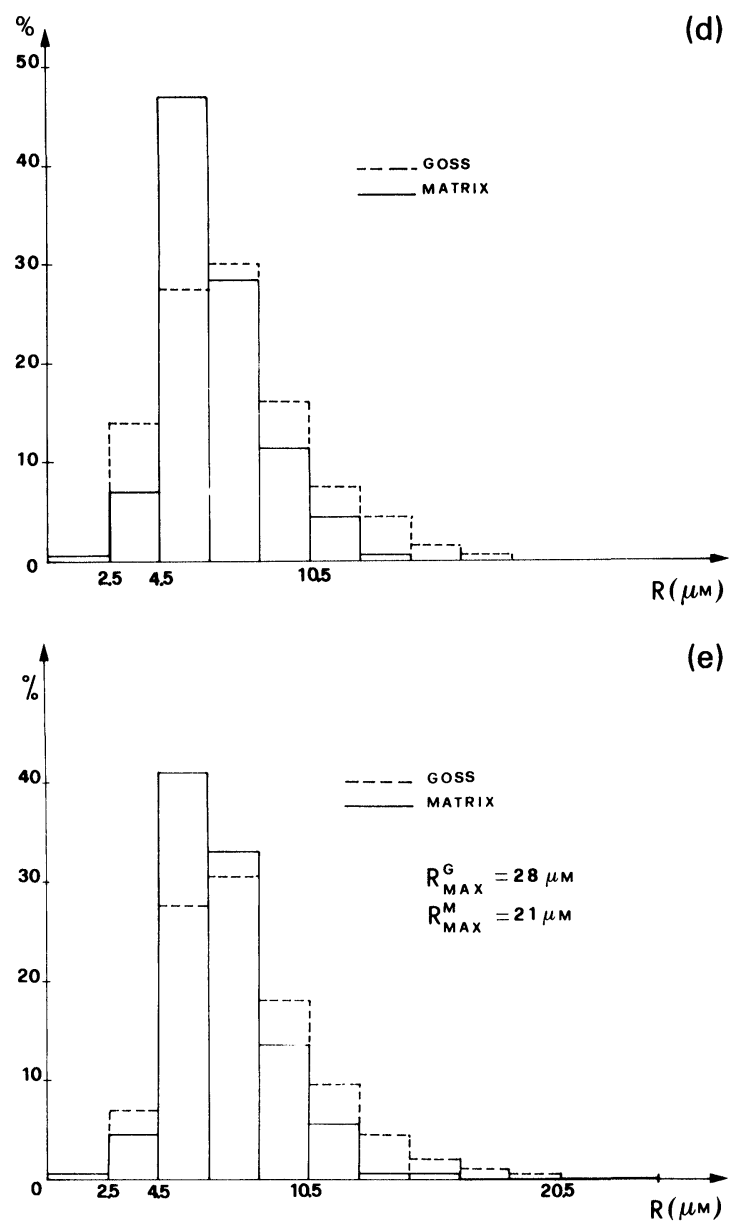

Figure 2 (d), (e) Computer simulated grain size distributions for Goss and matrix grains to be compared with those in Figure 2(b) and (c) respectively. The initial size distributions for the simulation were those of Figure 2(a) with an inhibition value $I_{z}=1400 \mathrm{~cm}^{-1}$.

(Abbruzzese and Fortunati, 1987) have shown that the Goss grains have not any size advantage after primary recrystallization and that the reason for the selection of Goss texture should be due to an alternative mechanism.

Figure $2 b$ and $2 c$ show the evolution of the grain size distributions 

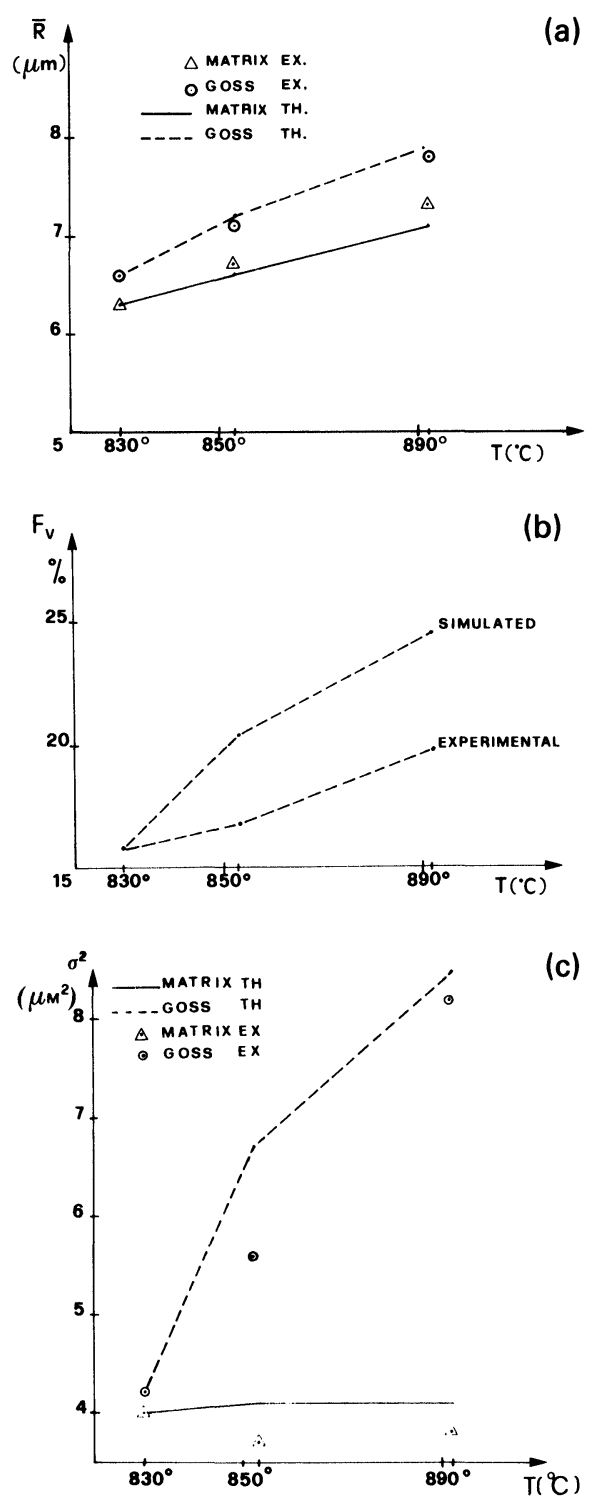

Figure 3 (a), (b), (c) Experimental (EX) data for the Goss and matrix mean radii $(\overline{\mathrm{R}})$, Goss volume fraction $\left(F_{v}\right)$ and size distribution variance $\left(\sigma^{2}\right)$ respectively in comparison with the computer simulated results $(\mathrm{TH})$. 
at different soaking temperatures $\left(853^{\circ}\right.$ and $\left.892^{\circ} \mathrm{C}\right)$. The increase of the mean radius of Goss grains is faster than that of their matrix and it corresponds to a widening of their size distribution especially as regards the development of a long tail in the distribution, that is the achievement of a size advantage for a certain number of grains in the system.

The characteristic reduction of the relative number of the smallest grains during the growth in presence of second phase particles (Abbruzzese 1985a, b, Abbruzzese and Ricci Bitti, 1984) is clearly shown for both distributions. Moreover this obvious phenomenon is responsible, together with the growth of the biggest grains, for the relative number increase in the largest size classes.

In Figure $3 a, b, c$ the experimental data for the mean radii, volume fraction and variance of the size distribution are shown.

It is worth noting that the variance of the Goss size distribution grows faster than that of the matrix due to the development of the long tail as already discussed above. Instead the variance of the matrix distribution remains constant or has a little drop before staying constant as it is usual for grain growth in presence of a strong enough inhibition from second phase particles (Abbruzzese, 1985).

\section{COMPUTER SIMULATIONS AND DISCUSSION}

The behaviour of the size distribution of Goss grains suggests that these grains should have special conditions in the system as regards the factors which influence the boundary mobility and/or the specific surface energy (Koh and Dunn, 1960, Dunn and MacHargue, 1960, Harase et al., 1986, Abbruzzese and Barisoni, 1986). For instance the typical texture present after primary recrystallization shows a certain Goss orientation in presence of a strong enough $\langle 111\rangle / /$ ND fiber; with some components belonging and close to this fiber the condition of a special orientation relationship (rotation of $20^{\circ}-35^{\circ}$ about $\langle 110\rangle$ common direction) is fulfilled obtaining the maximum velocity in grain boundary movement (Ibe and Lücke, 1966).

This general idea applied to Goss selection in silicon-iron was already discussed in other papers (Abbruzzese and Barisoni, 1986, 
Abbruzzese and Fortunati, 1987) and the particular grain growth process involved was simulated using the general statistical model for grain growth in presence of texture and second phase particles (Abbruzzese and Fortunati, 1987).

In this simulation by two texture components only a difference in grain boundary mobility was assumed, while the specific surface energy was kept constant irrespectively of the relative orientation between the grains.

The agreement with experimental results was quite good but the differences in mobilities between the two different boundaries had to be ten times of that used inside the single texture, to fit the experimental data.

Following the recent results (Harase et al., 1986), stating that Goss grains have the largest frequency of coincidence boundaries in silicon-iron, a computer simulation of the texture development was performed assuming that in the two texture components description, the Goss-matrix boundaries have a specific surface energy lower than those inside grains belonging to the matrix which contains all the other orientations $\left(\gamma^{\mathrm{GM}}=\gamma^{\mathrm{MG}}=350 \mathrm{erg} \mathrm{cm}^{-2}\right.$; $\gamma^{\mathrm{MM}}=500 \mathrm{erg} \mathrm{cm}^{-2}$ and $\gamma^{\mathrm{GG}}=250 \mathrm{erg} \mathrm{cm}^{-2} ; \mathrm{G}=\mathrm{GOSS} ; \mathrm{M}=$ MATRIX).

The boundary mobilities are here assumed constant so that it was possible to check whether differences in surface energy were already sufficient to explain the selection process. The inhibition value $I_{z}=1400 \mathrm{~cm}^{-1}$ used in the simulation was estimated from the $\{100\}\langle 001\rangle$ grain size distribution which was reasonably near the stagnation condition (see Figure 1) (Abbruzzese, 1985a, b).

The starting point for the computer simulation was the experimental size distributions measured for both Goss and matrix components and the numerical fraction of the Goss grains was assumed equal to that measured on the plane $(\sim 15 \%)$.

In Figure $2 \mathrm{~d}$ and $2 \mathrm{c}$ the simulations of the size distribution evolution for both texture components are shown and in Figure 3a, $\mathrm{b}$ and $\mathrm{c}$ the mean radii, the volume fractions and the size distribution variances are compared to the experimental results. The agreement is in general quite good especially as regards the evolution of the matrix distribution that is well described in almost all the details (it should be remarked that this was obtained with a good statistic analysis namely $\sim 1700$ grains): moreover the widen- 
ing of the Goss distribution (see also Figure 3c) and the evolution of the mean radii are in rather good agreement.

The theoretical change of volume fraction appears a little higher $(24 \%)$ than the experimental one $(20 \%)$ but this seems to be due to the sensitivity of this parameter in respect to the description by only two texture components and to the presence of a longer tail in the theoretical size distribution of the Goss grains.

It is also interesting to note that the O.D.F. calculation from $\mathrm{X}$-ray pole figure measurements was not able to give clear informations on the texture evolution at different temperatures and this might be due to the very small change (less than $5 \%$ ) in volume fraction involved which is comparable with the typical O.D.F. calculation errors.

In any case the incubation of secondary recrystallization in silicon-iron is fully described by a selective grain growth process which can be ascribed to differences in grain boundary energies and/or mobilities. The statistical theory of grain growth is able to describe completely and simulate the details of the process also using relatively rough assumptions as the presence of only two texture components in the system.

Further improvements of the theoretical description will be possible when more reliable data on the thermodynamical parameters of the boundaries (surface energy and mobility) as a function of the relative orientation relationships will be available.

\section{References}

Abbruzzese, G. (1985). Acta Met. 33, 1329.

Abbruzzese, G. (1985). Computer Simulation of Microstructural Evolution. Ed. D. J. Srolovitz, (Metallurgical Society of AIME, Warrandale), 61.

Abbruzzese, G. and Barisoni, M. (1986). Journal of Magnetism and Magnetic Materials 57, 1621.

Abbruzzese, G. and Ricci Bitti, R. (1984). Journal of Magnetism and Magnetic Materials 41, 11.

Abbruzzese, G. and Fortunati, S. (1987). Proceedings of SMM8 Conference, to be published in Physica Scripta.

Abbruzzese, G. and Lücke, K. (1986). Acta Met. 34, 905.

Abbruzzese, G. and Lücke, K. (1986). Proceedings of 7th Ris $\varnothing$ International Symposium on Metallurgy and Material Science, Roskilde, 1.

Abbruzzese, G. and Lücke, K. (1987). Proceedings of 8th International Conference on Texture of Materials, Sante Fe, to be published.

Beguinot, J. and Lesbats, C. (1977). Metallography 10, 111. 
Dunn, C. G. and Mac-Hargue, C. J. (1960). J. Appl. Phys. 31, 1767.

Harase, J. et al. (1986). Proceedings of 7th Ris $\varnothing$ International Symposium on Metallurgy and Material Science, Roskilde, 343.

Ibe, G. and Lücke, K. (1966). Recrystallization, Grain Growth and Texture, Ed. H. Margolin, 437.

Inokuti, Y. et al. (1981). Proceedings of 6th International Conference on Texture of Materials, Tokyo, 2, 948.

Koh, P. K. and Dunn, C. G. (1960). Trans. AIME 218, 65.

Matsuo, M. et al. (1981). Proceedings of 6th International Conference on Texture of Materials, Tokyo, 2, 918.

May, J. E. and Turnbull, D. (1958). Trans. AIME 212, 769.

Underwood, E. E. (1968). Quantitative Microscopy, eds. R. T. De Hoff and F. N. Rhines (McGraw-Hill, New York) p. 162. 